\title{
nbodykit: An Open-source, Massively Parallel Toolkit for Large-scale Structure
}

\author{
Nick Hand ${ }^{1,2}(1), Y_{1}$ Feng $^{2}$, Florian Beutler ${ }^{3,4}$, Yin Li ${ }^{2,4,5,6}$, Chirag Modi ${ }^{2,5}$, Uroš Seljak ${ }^{2,5}$, and Zachary Slepian ${ }^{2,4,7}$ \\ ${ }^{1}$ Astronomy Department, University of California, Berkeley, CA 94720, USA; nhand@berkeley.edu \\ ${ }^{2}$ Berkeley Center for Cosmological Physics, University of California, Berkeley, CA 94720, USA \\ ${ }^{3}$ Institute of Cosmology \& Gravitation, Dennis Sciama Building, University of Portsmouth, Portsmouth, PO1 3FX, UK \\ ${ }^{4}$ Lawrence Berkeley National Laboratory, 1 Cyclotron Road, Berkeley, CA 94720, USA \\ ${ }^{5}$ Physics Department, University of California, Berkeley, CA 94720, USA \\ ${ }^{6}$ Kavli Institute for the Physics and Mathematics of the Universe (WPI), UTIAS, The University of Tokyo, Chiba 277-8583, Japan \\ Received 2017 December 15; revised 2018 August 6; accepted 2018 August 14; published 2018 September 18
}

\begin{abstract}
We present nbodykit, an open-source, massively parallel Python toolkit for analyzing large-scale structure (LSS) data. Using Python bindings of the Message Passing Interface, we provide parallel implementations of many commonly used algorithms in LSS. nbodykit is both an interactive and scalable piece of scientific software, performing well in a supercomputing environment while still taking advantage of the interactive tools provided by the Python ecosystem. Existing functionality includes estimators of the power spectrum, two- and three-point correlation functions, a friends-of-friends grouping algorithm, mock catalog creation via the halo occupation distribution technique, and approximate $N$-body simulations via the FastPM scheme. The package also provides a set of distributed data containers, insulated from the algorithms themselves, that enables nbodykit to provide a unified treatment of both simulation and observational data sets. nbodykit can be easily deployed in a highperformance computing environment, overcoming some of the traditional difficulties of using Python on supercomputers. We provide performance benchmarks illustrating the scalability of the software. The modular, component-based approach of nbodykit allows researchers to easily build complex applications using its tools. The package is extensively documented at http://nbodykit.readthedocs.io, which also includes an interactive set of example recipes for new users to explore. As open-source software, we hope nbodykit provides a common framework for the community to use and develop in confronting the analysis challenges of future LSS surveys.
\end{abstract}

Key words: large-scale structure of universe - methods: data analysis - methods: numerical

\section{Introduction}

The analysis of large-scale structure (LSS) data sets has played a pivotal role in establishing the current concordance paradigm in modern cosmology, the $\Lambda$ CDM model. From the earliest galaxy surveys (Davis et al. 1985; Maddox et al. 1990), comparisons between the theoretical predictions for and observations of the distribution of matter in the universe have proven to be a valuable tool. Indeed, LSS observations, in combination with cosmic microwave background measurements, provided some of the earliest evidence for the $\Lambda \mathrm{CDM}$ model, e.g., Efstathiou et al. (1990), Krauss \& Turner (1995), and Ostriker \& Steinhardt (1995). Interest in LSS surveys increased immensely following the first direct evidence for cosmic acceleration (Riess et al. 1998; Perlmutter et al. 1999), as it was realized that the baryon acoustic oscillation (BAO) feature imprinted on large-scale clustering provided a "standard ruler" to map the expansion history (Eisenstein et al. 1998; Blake \& Glazebrook 2003; Seo \& Eisenstein 2003). From its first measurements (Cole et al. 2005; Eisenstein et al. 2005) to more recent studies (Font-Ribera et al. 2014; Delubac et al. 2015; Alam et al. 2017; Slepian et al. 2017), the BAO has proved to be a valuable probe of cosmic acceleration, enabling the most precise measurements of the expansion history of the universe over a wide redshift range. Analyses of these data sets have also pushed us closer to answering other important questions in contemporary cosmology, including deviations from general relativity (Mueller et al. 2018), the neutrino mass scale (Lesgourgues \& Pastor 2006; Beutler

\footnotetext{
Einstein Fellow.
}

et al. 2014), and the existence of primordial non-Gaussianity (Slosar et al. 2008; Desjacques \& Seljak 2010).

The foundations of the numerical methods used in LSS data analysis today go back several decades. Hockney \& Eastwood (1981) discussed several important computer simulation methods, including but not limited to mass assignment interpolation windows and the interlacing technique for reducing aliasing. The friends-of-friends (FOF) algorithm for identifying halos from a numerical simulation was first utilized in Davis et al. (1985). The most commonly used clustering estimators for the two-point correlation function (2PCF) and power spectrum were first developed in Landy \& Szalay (1993) and Feldman et al. (1994), respectively, and techniques to measure anisotropic clustering via a multipole basis were first used around the same time, e.g., Cole et al. (1995). Other modern, well-established numerical techniques include $N$-body simulation methods, e.g., Springel et al. (2001) and Springel (2005), and the use of KD trees in correlation function estimators (Moore et al. 2001).

Recent years have brought important updates to these analysis techniques. Advances in LSS observations, with increased sample sizes and statistical precision, have driven the development of new statistical estimators while also increasing modeling complexities and creating a need to reduce wall-clock times. Recently, we have seen faster power spectrum and 2PCF multipole estimators (Yamamoto et al. 2006; Bianchi et al. 2015; Scoccimarro 2015; Slepian \& Eisenstein 2015a, 2016; Hand et al. 2017a) and improved FOF algorithms (Springel 2005; Behroozi et al. 2013; Feng \& Modi 2017). Highly optimized software, e.g., TreeCorr (Jarvis et al. 2004) and Corrfunc (Sinha \& Garrison 2017), is 
also becoming increasingly common. New statistical estimators, e.g., Slepian \& Eisenstein (2015b, 2018) and Castorina \& White (2018), are being developed to extract as much information as possible from LSS surveys. The rise of particle mesh simulation methods (Merz et al. 2005; Tassev et al. 2013; White et al. 2014; Feng et al. 2016) has offered a computationally cheaper alternative to running full $\mathrm{N}$-body simulations. Finally, tools have emerged to help deal with the growing complexities of modeling the connection between halos and galaxies (Hearin et al. 2017). These examples represent just a sampling of the recent updates to LSS data analysis and modeling techniques.

The well-established foundation of LSS numerical methods suggests the community could benefit from a standard software package providing implementations of these methods. Such a package would also serve as a common framework for users as they incorporate future extensions and advancements. Given the already rising wall-clock times of current analyses and the expected volume of data from next-generation LSS surveys, scaling performance should also be a key priority.

Several computing trends in the past few years have emerged to help make such a software package possible. First, the Python programming language ${ }^{8}$ has emerged as the most popular language in the field of astronomy (Momcheva \& Tollerud 2015; NSF 2017), and the astropy ${ }^{9}$ package (Astropy Collaboration et al. 2013; The Astropy Collaboration et al. 2018) has led the development of an astronomy-focused Python ecosystem. Python's elegant syntax and dynamic nature make the language easy to learn and work with. Combined with its object-oriented focus and the larger ecosystem containing SciPy ${ }^{10}$ (Jones et al. 2001-2017), NumPy ${ }^{11}$ (van der Walt et al. 2011), IPython ${ }^{12}$ (Perez \& Granger 2007), and Jupyter $^{13}$ (Kluyver et al. 2016), Python is well suited for both rapid application development and use in scientific research. Second, the availability and performance of largescale computing resources continues to grow, and initiatives, e.g., The Exascale Computing Project, ${ }^{14}$ have been established to ensure the sustainability of this trend. At the same time, solutions to the traditional barriers to using Python on massively parallel, high-performance computing (HPC) machines have been developed. The mpi4py package (Dalcín et al. 2008; Dalcin et al. 2011) has facilitated the development of parallel Python applications by providing bindings of the Message Passing Interface (MPI) standard. Furthermore, tools have been developed, e.g., Feng \& Hand (2016), to alleviate the start-up bottleneck encountered when launching Python applications on HPC systems.

Motivated by these recent developments, we present the first public release of nbodykit (v0.3. $0^{15}$ ), an open-source, parallel toolkit written in Python for use in the analysis of LSS data. Designed for use on HPC machines, nbodykit includes fully parallel implementations of a canonical set of

\footnotetext{
8 http://python.org

9 http://www.astropy.org

${ }^{10}$ https://www.scipy.org

11 http://www.numpy.org

12 https://ipython.org

13 http://jupyter.org

14 https://www.exascaleproject.org

15 Version 0.3 .0 is archived with doi:10.5281/zenodo.1336768. Code development continued during the publication process, and the latest release is currently v0.3.4, archived with doi:10.5281/zenodo. 1336774 .
}

LSS algorithms. It also includes a set of distributed and extensible data containers, which can support a wide variety of data formats and large volumes of data. These data containers are insulated from the algorithms themselves, allowing nbodykit to be used for either simulation or observational data sets.

We have balanced the scalable nature of nbodykit with an object-oriented, component-based design that also facilitates interactive use. This allows researchers to take advantage of interactive Python tools, such as the Jupyter notebook, as well as integrate nbodykit components with their own software to build larger applications that solve specific problems in LSS. nbodykit has been designed to allow fast prototyping of analysis scripts in an interactive environment before deploying finalized workflows to an HPC cluster. In the future, we expect tools that connect these steps, e.g., Parsl ${ }^{16}$ and ipyparallel, ${ }^{17}$ to become commonplace, allowing workflows to be fully contained in an interactive environment.

nbodykit has been developed, tested, and deployed on the Edison and Cori Cray supercomputers at the National Energy Research Scientific Computing Center (NERSC) and has been utilized in several published research studies (Feng et al. 2016; Waters et al. 2016; Hand et al. 2017a, 2017b; Modi et al. 2017; Pinol et al. 2017; Schmittfull et al. 2017; Ding et al. 2018). Since its start, it has been developed on GitHub as open-source software at https://github.com/bccp/nbodykit.

The objective of this paper is to provide an overview of the nbodykit software and familiarize the community with some of its capabilities. We hope that researchers find nbodykit to be a useful tool in their scientific work and in the spirit of open science, and that it continues to grow via community contributions. Extensive documentation and tutorials are available at http://nbodykit.readthedocs.io, and we do not aim to provide such detailed documentation in this work. The documentation also includes instructions for launching an interactive environment containing a set of example recipes. This allows new users to explore nbodykit without setting up their own nbodykit installation.

The paper is organized as follows. We provide a broad overview of nbodykit in Section 2 and discuss a more detailed list of its capabilities in Section 3. We describe our development process and deployment strategy for nbodykit in Section 4. Section 5 presents an illustrative example use case, and Section 6 outlines performance benchmarks for various algorithms. Finally, we conclude and summarize in Section 7.

\section{Overview}

\subsection{Initializing nbodykit}

A core design goal of nbodykit is maintaining an interactive user experience, allowing the user to quickly experiment and to prototype new analysis pipelines while still leveraging the power of parallel processing when necessary. We adopt a "lab" framework for nbodykit, where all of the necessary data containers and algorithms can be imported from the nbodykit.lab module. Furthermore, we utilize Python's logging module to print messages at runtime, which allows users to track the progress of the application in real time. Typically, applications using nbodykit begin with the following statements.

\footnotetext{
16 parsl.readthedocs.io

17 https://github.com/ipython/ipyparallel
} 


\subsection{The nbodykit Ecosystem}

nbodykit is explicitly maintained as a pure Python package. However, it depends on several compiled extension packages that each focus on more specialized tasks. This approach enables nbodykit to describe higher-level abstractions in Python and retains the readability, syntax, and user interface benefits of the Python language. For computationally expensive sections of the code base, we use the compiled extension packages for speed. With the emergence of Python package managers such as Anaconda, ${ }^{18}$ the availability of binary versions of these compiled packages for different operating systems has sufficiently eased most installation issues in our experience (see Section 4.3).

Below, we describe some of the more important dependencies of nbodykit, each of which is focused on solving a particular problem:

1. pfft-python: a Python binding of the PFFT software (Pippig 2013), which computes parallel fast Fourier transforms (FFTs; Feng 2017a).

2. pmesh: particle mesh calculations, including density field interpolation and discrete parallel FFTs via pfftpython (Feng 2017b).

3. bigfile: a reproducible, massively parallel input/output (IO) library for large, hierarchical data sets (Feng 2017c).

4. kdcount: spatial indexing operations via KD trees (Feng 2017d).

5. classylss: a Python binding of the CLASS Boltzmann solver (Hand \& Feng 2017).

6. fastpm-python: a Python implementation of the FastPM scheme for quasi $N$-body simulations (Feng et al. 2016; Feng 2017e).

7. Corrfunc: a set of high-performance routines for computing pair-counting statistics (Sinha \& Garrison 2017).

8. Halotools: a package to build and test models of the galaxy-halo connection (Hearin et al. 2017).

\subsection{A Component-based Approach}

The design of nbodykit focuses on a modular, component-based approach. The components are exposed to the user as a set of Python classes and functions, and users can combine these components to build their specific applications. This design differs from the more commonly used alternative in cosmology software, which is a monolithic application controlled by a single configuration file, e.g., as in $\mathrm{CAMB}$ (Lewis et al. 2000), CLASS (Blas et al. 2011), and Gadget (Springel et al. 2001). We note that modular, object-oriented designs using Python have become more popular recently, e.g., astropy, the yt project (Turk et al. 2011), Halotools (Hearin et al. 2017), and Colossus (Diemer 2017). During the development process, we have found that a component-based approach offers greater freedom and flexibility to build complex applications with nbodykit.

We present some of the main classes and interfaces and how data flows through them in Figure 1. In the subsections to follow, we will provide an overview of some of the components outlined in this figure.

\footnotetext{
18 https://anaconda.com
}

\subsubsection{Catalog}

A Catalog is a Python object derived from a CatalogSource class that holds information about discrete objects ${ }^{19}$ in a column-based format. Catalogs implement a random-read interface, which allows users to access arbitrary slices of the data while also taking advantage of the high throughput of a parallel file system. Often, users will initialize Catalog objects by reading data from a file on disk, using a NumPy array already stored in memory, or by generating simulated particles at runtime using one of nbodykit's built-in classes.

\subsubsection{Mesh}

A Mesh is a Python object that computes a discrete representation of a continuous quantity on a uniform mesh. It is derived from a MeshSource class and provides a paintable interface, which refers to the process of "painting" the density field values onto the discrete mesh cells. When the user calls the paint () function, the mesh data is returned as a threedimensional array. Mesh objects can be created directly from a Catalog via the to_mesh() function or by generating simulated fields directly on the mesh.

\subsubsection{Algorithms}

Algorithms are implemented as Python classes and interact with data by consuming Catalog and Mesh objects as input. The algorithm is executed when the user initializes the class, and the returned instance stores the results as attributes.

\subsubsection{Serialization and Reproducibility}

Most objects in nbodykit are serializable ${ }^{20}$ via a save () function. Algorithm classes not only save the result of the algorithm but also save input parameters and metadata. They typically implement both a save() and load() function, such that the algorithm result can be de-serialized into an object of the same type. The two main data containers, catalogs and meshes, can be serialized using nbodykit's intrinsic format, which relies on the massively parallel IO library bigfile (Feng 2017c). nbodykit includes support for reading these serialized results from disk back into Catalog or Mesh objects.

\subsection{Parallelism}

\subsubsection{Data-based}

nbodykit is fully parallelized using the Python bindings of the MPI standard available through mpi4py. The MPI standard allows processes running in parallel, each with their own memory, to exchange messages. This mechanism enables independent results to be computed by individual processes and then combined into a single result.

Both the Catalog and Mesh objects are distributed data containers, meaning that the data are spread out evenly across the available processes within an MPI communicator. ${ }^{21}$ Nearly all algorithm calculations are performed on this distributed

\footnotetext{
19 Here, "object" can represent galaxies, simulation particles, mass elements, etc.

${ }^{20}$ Serialization (and its reverse, de-serialization) refers to the process of storing a Python object on disk in a format such that it can be reconstructed at a later time.

21 The MPI communicator is responsible for managing the communication between a set of parallel processes.
} 


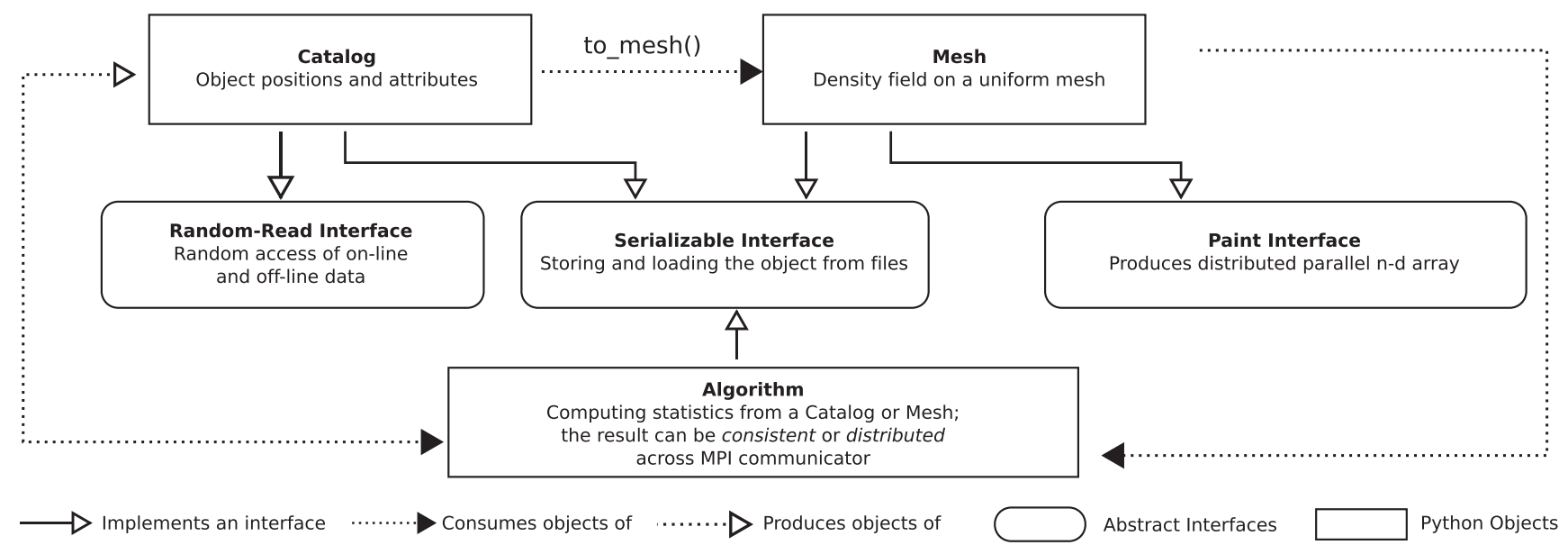

Figure 1. The components and interfaces of nbodykit. The main Python classes are Catalog, Mesh, and Algorithm objects, which are described in more detail in Section 2.3. Algorithm results can be consistent, where all processes hold the same data, or distributed, where data are spread out evenly across parallel processes.

data, with final results computed via a reduce operation across all processes in the communicator. Rarely throughout the code base, data are instead gathered to a single root process, and operations are performed on these data before re-distributing the results to all processes. This only occurs when wall-clock time will not be a concern for most use cases and the additional complexity of a massively parallel implementation is not merited.

The distributed nature of the Catalog object is implemented by using the random-read interface to access different slices of the tabular data for different processes. The values of a Mesh object are stored internally on a three-dimensional NumPy array, which is distributed evenly across all processes. The domain of the 3D mesh is decomposed across parallel processes using the particle mesh library pmesh, which also provides an interface for computing parallel FFTs of the mesh data using pfft-python. The pfft-python software exhibits excellent scaling with the number of available processes, enabling high-resolution (large number of cells) mesh calculations.

\subsubsection{Task-based}

The analysis of LSS data often involves hundreds to thousands of repetitions of a single, less computationally expensive task. Examples include estimating the covariance matrix of a clustering statistic from a set of simulations and best-fit parameter estimation for a model. nbodykit implements a TaskManager utility to allow users to easily iterate over multiple tasks while executing in parallel. Users can specify the desired number of processes assigned to each task, and the TaskManager will iterate through the tasks, ensuring that all processes are being utilized.

\section{Capabilities}

In this section, we provide a more detailed overview of some of the main components of nbodykit. In particular, we describe how cosmology calculations are performed (Section 3.1), outline the available Catalog (Section 3.2) and Mesh (Section 3.3) classes, and provide details and references for the various algorithms currently implemented (Section 3.4).

\subsection{Cosmology}

The nbodykit.cosmology module includes functionality for representing cosmological parameter sets and computing various common theoretical quantities in LSS that depend on the background cosmological model. The underlying engine for these calculations is the CLASS Boltzmann solver (Blas et al. 2011; Lesgourgues 2011). We use the Python bindings of the CLASS C library provided by the classylss package. Comparing to the binding provided by the CLASS source code, classylss is a direct mapping of the CLASS object model to Python and integrates with the NumPy array protocol natively.

The main object in the module is the Cosmology class, which users can initialize by specifying a unique set of cosmological parameters (using the syntax of CLASS). This class represents the background cosmological model and contains methods to compute quantities that depend on the model. Most of the CLASS functionality is available through methods of the Cosmology object. Examples include distance as a function of redshift $z$, the Hubble parameter $H(z)$, the linear power spectrum, the nonlinear power spectrum, and the density and velocity transfer functions. Several Cosmology objects are provided for well-known parameter sets, including the WMAP 5, 7, and 9 year results (Komatsu et al. 2009, 2011; Hinshaw et al. 2013), and the Planck 2013 and 2015 results (Planck Collaboration et al. 2014, 2016).

The nbodykit. cosmology module also includes classes to represent theoretical power spectra and correlation functions. The LinearPower class can compute the linear power spectrum as a function of redshift and wavenumber, using either the transfer function as computed by CLASS or the analytic approximations of Eisenstein \& Hu (1999). The latter includes the so-called "no-wiggle" transfer function, which includes no BAO but the correct broadband features, and is useful for quantifying the significance of potential BAO features. Similarly, we provide the NonlinearPower object to compute nonlinear power spectra, using the Halofit implementation in CLASS (Smith et al. 2003), which includes corrections from Takahashi et al. (2012). The ZeldovichPower class uses the linear power spectrum object to compute the power spectrum in the Zel'dovich approximation (tree-level Lagrangian perturbation theory). The implementation closely follows the appendices of Vlah et al. (2015) and relies on a 
Python implementation and generalization of the FFTLog algorithm $^{22}$ (Hamilton 2000). Finally, we also provide a Correlationfunction object to compute theoretical correlation functions from any of the available power classes (using FFTLog to compute the Fourier transform).

We choose to use the CLASS software for the cosmological engine in nbodykit rather than the most likely alternative, the astropy. cosmology module. This allows nbodykit to leverage the full power of a Boltzmann solver for LSS calculations. We provide syntax compatibility between the Cosmology class and astropy when appropriate as well as functions to transform between the cosmology classes used by the two packages. However, we note that there are important differences between the two implementations. In particular, the treatment of massive neutrinos differs, with astropy using the approximations of Komatsu et al. (2011) rather than the direct calculations, as in CLASS.

\subsection{Catalogs}

In this section, we describe the two main ways that catalogs are created in nbodykit, as well as tools for cleaning and manipulating data stored in Catalog objects.

\subsubsection{Reading Data from Disk}

We provide support for loading data from disk into Catalog objects for several of the most common data storage formats in LSS data analysis. These formats include plaintext commaseparated value (CSV) data (via pandas; McKinney 2010), binary data stored in a column-based format, HDF5 data (via h5py; Collette 2017), FITS data (via fitsio; Sheldon 2017), and the bigfile data format. We also provide more specialized readers for particle data from the Tree-PM simulations of White (2002) and the legacy binary format of the GADGET simulations (Springel 2005). These Catalog objects use the nbodykit.io module, which includes several "file-like" classes for reading data from disk. These file-like objects implement a read () function that provides the random-read interface which returns a slice of the data for the requested columns. Users can easily support custom file formats by implementing their own subclass and read() interface.

Formats storing data on disk in a column-based structure yield the best performance results, as the entirety of the data do not need to be parsed to yield the desired slice of data on a given process. This is not true for the CSV storage format. We mitigate performance issues by implementing an enhanced version of the CSV parser in pandas that supports faster parallel random access. Our preferred IO format, bigfile, is massively parallel and stores data in a column-based format.

Finally, the Catalog object supports loading data from multiple files at once, providing a continuous view of the entirety of the data. This becomes particularly powerful when combined with the random-read interface, as arbitrary slices of the combined data can be accessed. For example, a single Catalog object can provide access to arbitrary slices of the output binary snapshots from an $N$-body simulation (stored over multiple files), often totaling $10-100 \mathrm{~GB}$ in size.

\footnotetext{
${ }^{22}$ https://github.com/eelregit/mcfit
}

\subsubsection{Generating Catalogs at Runtime}

nbodykit includes several Catalog classes that generate simulated data at runtime. The simplest of these allows generating random columns of data in parallel using the numpy. random module. We also provide a UniformCatalog class that generates uniformly distributed particles in a box. These classes are useful for testing purposes, as well as for use as unclustered, synthetic data in clustering estimators.

nbodykit also includes functionality for generating more realistic approximations of LSS. LogNormalCatalog generates a set of objects by Poisson sampling a log-normal density field and applies the Zel'dovich approximation to model nonlinear evolution (Coles \& Jones 1991; Agrawal et al. 2017). The user can specify the input linear power spectrum and the desired output redshift of the catalog.

Catalog objects can also be created using the mock generation techniques of the Halotools software (Hearin et al. 2017) for populating halos with objects. Halotools includes functionality for populating halos via a wide range of techniques, including the halo occupation distribution (HOD), conditional luminosity function, and abundance matching methods. We refer the reader to Hearin et al. (2017) for further details. nbodykit supports using a generic Halotools model to populate a halo catalog. We also include built-in, specialized support for the HOD models of Zheng et al. (2007), Leauthaud et al. (2011), and Hearin et al. (2016).

Finally, the fastpm-python package implements an nbodykit Catalog object that generates particles via the FastPM approximate $N$-body simulation scheme (Feng et al. 2016). The FastPM library is massively parallel and exhibits excellent strong scaling with the number of available processes (see Section 6).

\subsubsection{On-demand Data Cleaning}

nbodykit uses the dask library (Dask Development Team 2016) to represent the data columns of a Catalog object as dask array objects instead of using the more familiar NumPy array. The dask array has two key features that help users work interactively with data and, in particular, large data sets. The first feature is delayed evaluation. When manipulating a dask array, operations are not evaluated immediately but instead stored in a task graph. Users can explicitly evaluate the dask array (returning a NumPy array) via a call to a compute () function. Second, dask arrays are chunked. The array object is internally divided into many smaller arrays, and calculations are performed on these smaller "chunks."

The delayed evaluation of dask arrays is particularly useful during the process of data cleaning, when users manipulate input data before feeding it into the analysis pipeline. Common examples of data cleaning include changing the coordinate system from galactic to Euclidean, converting between unit conventions, and applying masks. When using large data sets, the time to load the full data set into memory can be significant. This delay hinders data exploration and limits the interactive benefits of the Python language. dask arrays allow users to design data-cleaning pipelines on the fly. If the data format on disk supports random-read access, users can easily select and peek at a small subset of data without reading the full data set. This becomes especially useful when prototyping scientific models in an interactive environment, such as a Jupyter notebook. 
The chunked nature of the dask array allows array computations to be performed on large data sets that do not fit into memory because the chunk size defines the amount of data loaded into memory at any given time. It effectively extends the maximum size of usable data sets from the size of the memory to the size of the disk storage. This feature also simplifies the process of dealing with large data sets in interactive environments.

\subsection{Meshes}

\subsubsection{Painting a Mesh}

The Mesh object implements a paint () function, which is responsible for generating the field values on the mesh and returning an array-like object to the user. Meshes provide an equal treatment of configuration and Fourier space, and users can specify whether the painted array is defined in configuration or Fourier space. In the former case, a RealField is returned and in the latter, a ComplexField. These objects are implemented by the pmesh package and are subclasses of the NumPy ndarray class. They are related via a real-tocomplex parallel FFT operation, implemented using pfftpython via the $r 2 \mathrm{c}()$ and $\mathrm{c} 2 \mathrm{r}(\mathrm{l})$ functions.

The paint () function paints mass-weighted (or equivalently, number-weighted) quantities to the mesh. The field that is painted is

$$
F(\boldsymbol{x})=\left[1+\delta^{\prime}(\boldsymbol{x})\right] V(\boldsymbol{x}),
$$

where $V(\boldsymbol{x})$ represents the field value painted to the mesh, and $\delta^{\prime}(\boldsymbol{x})=n^{\prime}(\boldsymbol{x}) / \bar{n}^{\prime}-1$ is the weighted overdensity field. It is related to the unweighted number density as $n^{\prime}(\boldsymbol{x})=W(\boldsymbol{x}) n(\boldsymbol{x})$, where $W(\boldsymbol{x})$ are the weights.

In nbodykit, users can control the behavior of both $V(\boldsymbol{x})$ and $W(\boldsymbol{x})$. In the default case, both quantities are unity, and the field painted to the mesh is $1+\delta$. As an illustration, $V(\boldsymbol{x})$ can be specified as a velocity component to paint the momentum field (mass-weighted velocity). We also provide a mechanism by which users can further transform the painted field on the mesh. The apply ( ) function can be used to apply a function to the mesh, either in configuration or in Fourier space. Multiple functions can be applied to the mesh, and the operations are performed when paint () is called.

\subsubsection{From Catalog to Mesh}

All Catalog objects include a to_mesh () function, which creates a Mesh object using the specified number of cells per mesh side. This function allows users to configure exactly how the catalog is interpolated onto the mesh. Users can choose from several different mass assignment windows, including the Cloud-In-Cell (CIC), Triangular Shaped Cloud (TSC), and Piecewise Cubic Spline (PCS) schemes (Hockney \& Eastwood 1981). The Daubechies wavelet (Daubechies 1992) and its symmetric counterpart ("Symlets;" see, e.g., PyWavelet $\mathrm{s}^{23}$ ) are also available. By default, the CIC window is used. The interlacing technique (Hockney \& Eastwood 1981; Sefusatti et al. 2016) can reduce the effects of aliasing in Fourier space. In this scheme, the Catalog object is interpolated onto two separate meshes separated by half of a cell size. When the fields are combined in Fourier space, the leading-order contribution to aliasing is eliminated.

\footnotetext{
${ }^{23}$ https://pywavelets.readthedocs.io
}

Users can also configure whether or not the window is compensated, which divides the density field in Fourier space by (Hockney \& Eastwood 1981)

$$
W(\boldsymbol{k})=\Pi_{i}\left[\operatorname{sinc}\left(\pi k_{i} / 2 k_{\mathrm{N}}\right)\right]^{p},
$$

where $i \in\{x, y, z\} ; p=2,3,4$ for CIC, TSC, and PCS, respectively; and $\operatorname{sinc}(x) \equiv \sin (x) / x$. The Nyquist frequency of the mesh is given by $k_{\mathrm{N}}=\pi N / L$, where $L$ is the box size, and $N$ is the number of cells per box side.

We provide comparisons of the various interpolation windows and correction methods in this section. First, Figure 2 illustrates the effects of interlacing when using the CIC, TSC, and PCS schemes. This comparison is similar to the detailed analysis presented in Sefusatti et al. (2016). Second, we show the effectiveness of the wavelet windows at reducing aliasing in Figure 3. For both figures, we paint a LogNormalcatalog of $5 \times 10^{7}$ objects to a mesh of $512^{3}$ cells in a box of side length $2500 \mathrm{~h}^{-1} \mathrm{Mpc}$. We compare the measured power spectrum to a "reference" power spectrum, computed using a mesh of $1024^{3}$ cells and the PCS window. When using the CIC, TSC, and PCS windows, we de-convolve the interpolation window using Equation (2), while we apply no such corrections when using wavelet-based windows.

Figure 2 confirms the results of Sefusatti et al. (2016) - the interlacing technique performs very well at reducing the effects of aliasing on the measured power spectrum. We achieve subpercent accuracy up to the Nyquist frequency when combining interlacing with the CIC, TSC, and PCS windows. In general, higher order windows perform better, with the PCS scheme achieving a precision of at least $\sim 10^{-5}$ up to the Nyquist frequency.

Figure 3 compares the performance of the Daubechies and Symlet wavelets to the CIC, TSC, and PCS windows. As in Figure 2, we plot the ratio of the power spectrum computed using meshes of size $512^{3}$ and $1024^{3}$ cells. We apply Equation (2) for the CIC, TSC, and PCS windows but do not apply any corrections when using the wavelet windows. For this comparison, we do not use interlacing. We are able to confirm the results of Cui et al. (2008) and Yang et al. (2009), which claim $2 \%$ accuracy on the power spectrum up to $k \approx 0.7 k_{\mathrm{N}}$ when using the DB6 window without any additional corrections. However, the wavelet windows fail to match the precision achieved when using interlacing, even when using the largest wavelet size tested here $(a=20)$. Furthermore, the Daubechies windows introduce scale dependence on large scales due to symmetry breaking (see the inset of Figure 3). The symmetric Symlet wavelets do not suffer from this issue but also cannot match the accuracy achieved when using interlacing.

Figure 3 also displays the relative speeds of each of the windows discussed in this section (bottom panel). These timing tests were performed using 64 cores on the NERSC Cori Phase I system. The wavelet windows are all significantly slower than the CIC, TSC, and PCS windows. The TSC and PCS methods are only marginally slower than the default CIC scheme, with slowdowns of $\sim 10 \%$ and $\sim 40 \%$, respectively. The $\mathrm{CIC}$, TSC, and PCS windows rely on optimized implementations in pmesh, while the wavelet windows use a slower lookup table implementation. Due to the precision of the interlacing technique and the relative speed of the TSC and PCS windows, we recommend using these options in most instances. However, it is generally best 


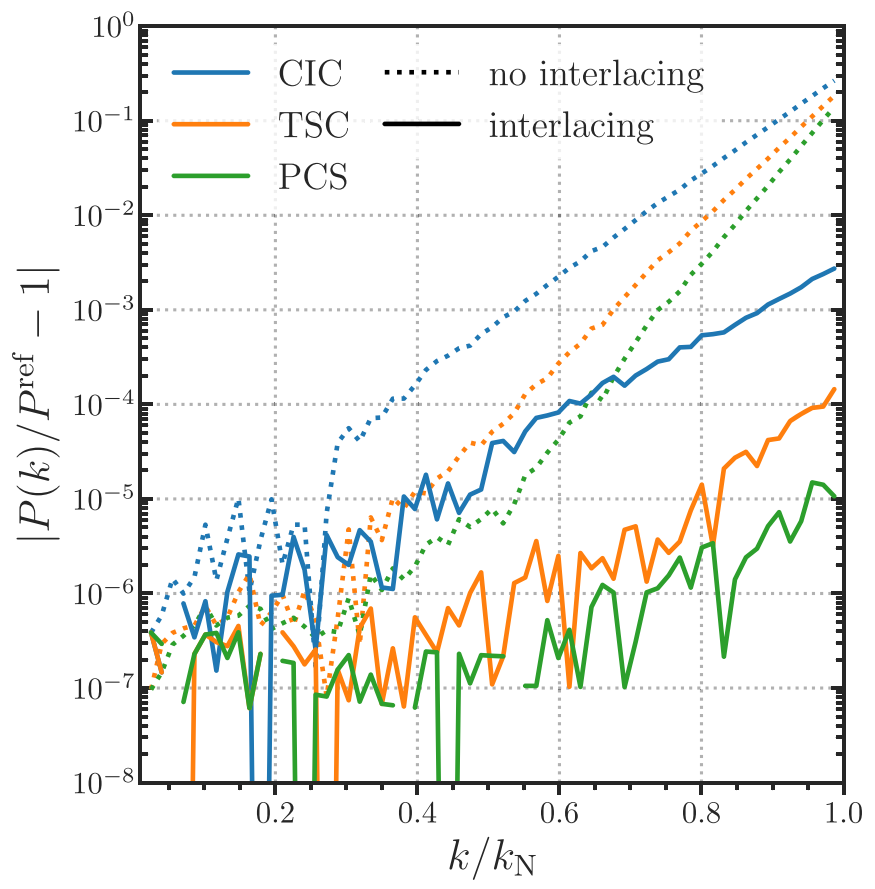

Figure 2. A comparison of the effects of interlacing when using the CIC, TSC, and PCS windows. We show the ratio of the power spectrum computed for a log-normal density field using a mesh with $512^{3}$ cells to a reference power spectrum $P^{\text {ref }}$, computed using a mesh with $1024^{3}$ cells. The ratio is shown as a function of wavenumber in units of the Nyquist frequency of the lower resolution mesh. In all cases, the appropriate window compensation is performed using Equation (2).
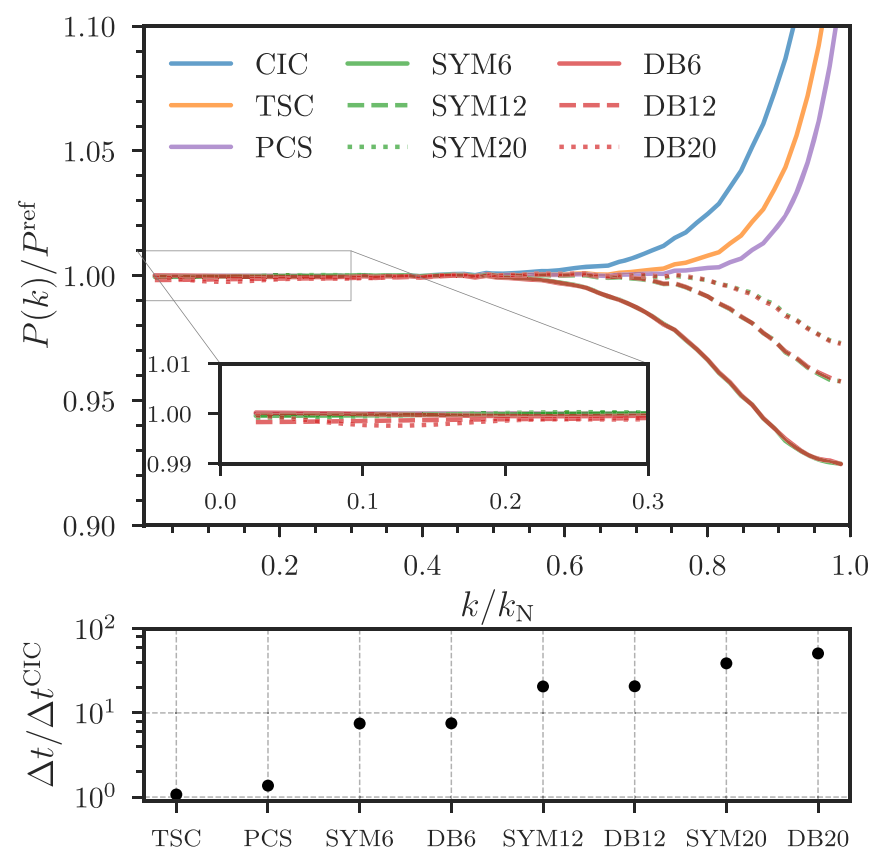

Figure 3. The performance of the Daubechies and Symlet wavelets in comparison to the CIC, TSC, and PCS windows. Wavelet windows of sizes $a=6,12$, and 20 are shown. Top: the ratio of the measured power to the reference power spectrum, as in Figure 2. Here, we apply no corrections when using the wavelet windows and apply Equation (2) for the CIC, TSC, and PCS windows. No interlacing is used for this test. Bottom: the speed of each interpolation window, relative to the CIC window. Speeds were recorded when computing the power spectra in the top panel.

to determine the optimal set of parameters for a particular application by running convergence tests with different parameter configurations.

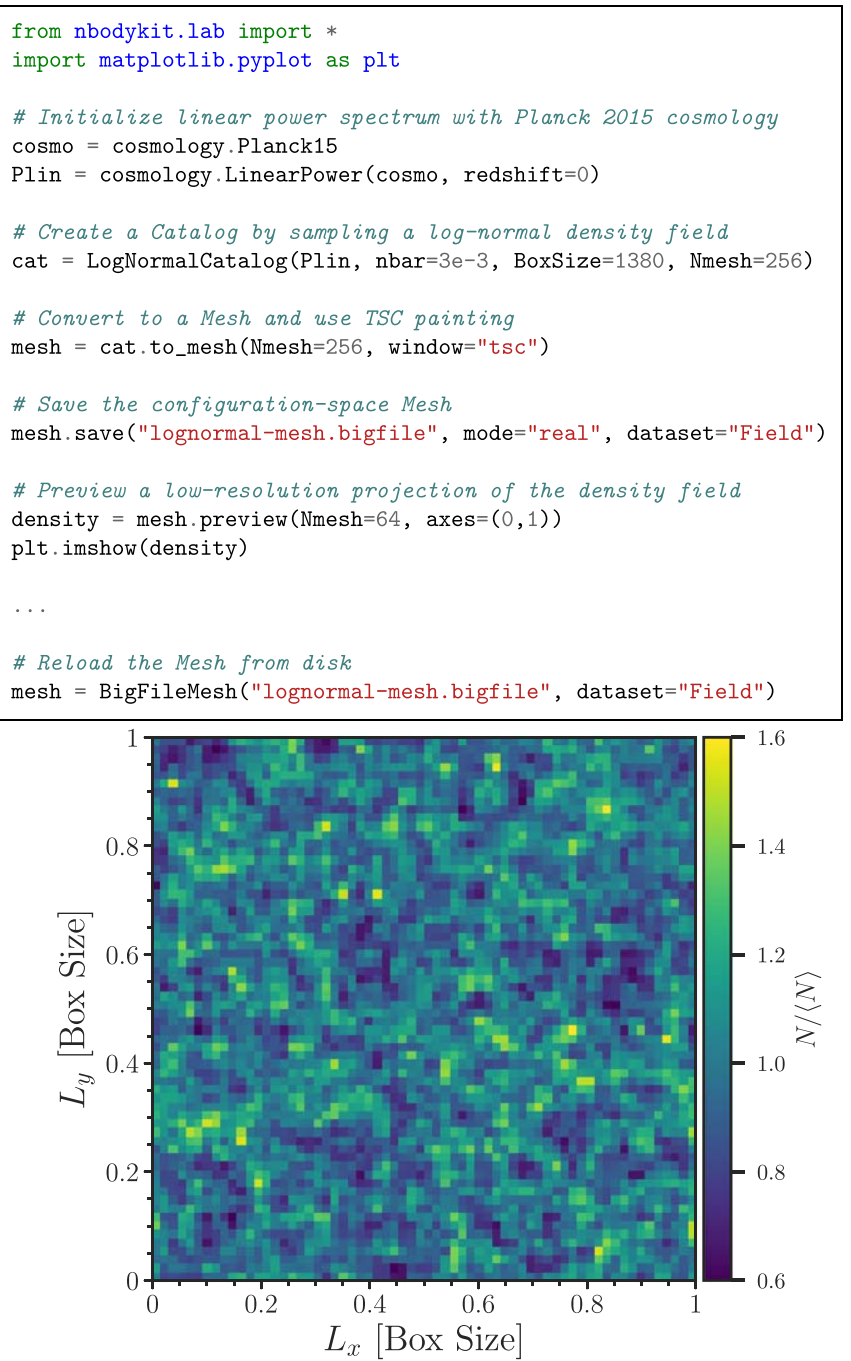

Figure 4. Top: an analysis pipeline illustrating the creation of a Mesh object from a Catalog, as well as how to serialize the painted mesh to disk and preview a low-resolution projection of the density field for inspection. Bottom: the two-dimensional, low-resolution preview of the painted density field $N /\langle N\rangle=1+\delta$.

\subsubsection{An Illustrative Example}

We demonstrate the use of Mesh objects through an example in Figure 4, which gives a short code snippet that creates a Mesh object from an existing Catalog, saves the configuration space density field to disk, and then reloads the data into memory. The snippet also demonstrates the preview () function, which can create a lower resolution projection of the full mesh field. This can be useful to quickly inspect mesh fields interactively, which would otherwise be difficult due to memory limitations. We show the preview of the density field from a log-normal catalog in the bottom panel of Figure 4, where the LSS is clearly evident, even in the low-resolution projection.

\subsection{Algorithms}

The nbodykit.algorithms module includes parallel implementations of some of the most commonly used LSS analysis algorithms. We take care to provide support for data sets from both observational surveys and $N$-body simulations. In this section, we provide an overview of the available 
functionality. The set of algorithms currently implemented is not meant to be exhaustive, but instead a solid foundation for LSS data analysis.

\subsubsection{Power Spectra}

For simulation boxes with periodic boundary conditions, the FFTPOwer algorithm measures the power directly from the square of the Fourier modes of the overdensity field. The 1D or 2D power spectrum, $P(k)$ or $P(k, \mu)$, can be computed, as well as the power spectrum multipoles $P_{\ell}(k)$. Here, $\mu$ represents the angle cosine between the pair separation vector and the line of sight. For observational data, in the form of R.A., decl., and redshift, the power spectrum multipoles of the density field can be computed using the ConvolvedFFTPower algorithm. The output of this algorithm can be accurately modeled using a theoretical power spectrum convolved with the survey window function (see, e.g., Beutler et al. 2017; Hand et al. 2017b). The implementation uses the FFT-based estimator described in Hand et al. (2017a), which requires $2 \ell+1$ FFTs to compute a given multipole of order $\ell$. This estimator improves the FFTbased estimator presented by Bianchi et al. (2015) and Scoccimarro (2015), building on the ideas of previous power spectrum estimators (Feldman et al. 1994; Yamamoto et al. 2006) and in particular, the treatment of the anisotropic 2PCF using spherical harmonics of Slepian \& Eisenstein (2015b). We also provide ProjectedFFTPower for computing the power spectrum of a field in a simulation box, projected along the specified axes. Such an observable can be useful for, e.g., Ly $\alpha$ or weak lensing data analysis (although modifications must be made for realistic cases beyond its current idealized form). The correctness of these algorithms has been verified using independent implementations from within the Baryon Oscillation Spectroscopic Survey (BOSS) collaboration.

\subsubsection{Two-point Correlation Functions}

nbodykit includes functionality for counting pairs of objects and computing their correlation function in configuration space. We leverage the blazing speed ${ }^{24}$ of the publicly available Corrfunc chaining mesh code for these calculations (Sinha \& Garrison 2017). We adapt its highly optimized pair-counting routines to perform calculations using MPI. We perform a domain decomposition on the input data such that the objects on a particular MPI rank are spatially confined to include all pairs within the maximum separation. For nonuniform density fields, the domain decomposition results in a particle load that is balanced across MPI ranks. ${ }^{25}$ The relevant pair-counting algorithms are SimulationBoxPairCount and SurveyDataPairCount. These classes can count pairs of objects as a function of the 3D separation $r$, the separation $r$ and angle to the line of sight $\mu$, the angular separation $\theta$, and the projected distances perpendicular $r_{p}$ and parallel $\pi$ to the line of sight.

Users can compute the correlation function of a Catalog using the SimulationBox2PCF and SurveyData2PCFclasses, which internally rely on the previously described paircounting classes. For data with periodic boundary conditions,

\footnotetext{
${ }^{24}$ Our benchmarks indicate that the pair-counting routines in Corrfunc are at least twice as fast as other publicly available codes, including TreeCorr, kdcount, Halotools, and SciPy KDTree.

${ }^{25}$ We thank Biwei Dai for the implementation of the load balancer.
}

we use analytic randoms to estimate the correlation function using the so-called "natural" estimator: $D D / R R-1$. A Catalog object holding synthetic randoms can be supplied, in which case the Landy-Szalay estimator (Landy \& Szalay 1993) is employed: $(D D-2 D R+R R) / R R$. The variations of the correlation function that can be computed by these two classes are as follows:

1. as a function of three-dimensional separation, $\xi(r)$;

2. accounting for the angle to the line of sight, $\xi(r, \mu)$ and $\xi\left(r_{p}, \pi\right)$;

3. as a function of angular separation, $w(\theta)$;

4. projected over the line-of-sight separations, $w_{p}\left(r_{p}\right)$.

The correctness of the pair-counting and correlation function algorithms described here was independently verified using the kdcount and Halotools software.

\subsubsection{Three-point Correlation Function (3PCF)}

The SimulationBox3PCF and SurveyData3PCF classes compute the multipoles of the isotropic 3PCF in configuration space. The algorithm follows the implementation described in Slepian \& Eisenstein (2015b), which scales as $\mathcal{O}\left(N^{2}\right)$, where $N$ is the number of objects. Their improved estimator relies on a spherical harmonic decomposition to achieve a similar scaling with $N$ as two-point clustering estimators. We note that the FFT-based implementation of this algorithm (presented in Slepian \& Eisenstein 2016) and the anisotropic version described in Slepian \& Eisenstein (2018) have not yet been implemented, although there are plans to do so in the future. We have verified the accuracy of the isotropic 3PCF classes against the implementation used in Slepian \& Eisenstein (2015b). An implementation of this algorithm including anisotropy written in $\mathrm{C}++$ and optimized for HPC machines was recently presented in Friesen et al. (2017).

\subsubsection{Grouping Methods}

The FOF class implements the well-known FOF algorithm, which identifies clusters of points that are spatially less distant than a threshold linking length. It uses a parallel implementation of the algorithm described in Feng \& Modi (2017), which utilizes KD trees and the kdcount software. FOF groups can be identified as a function of three-dimensional or angular separation. We also provide functions for transforming the output of the FOF algorithm to a Catalog of halo objects (a HaloCatalog) in a manner compatible with the Halotools software. While the FOF algorithm is commonly used in LSS analysis, it can lead to spurious artifacts; see, for example, Everitt et al. (2009) and Jain et al. (2004).

nbodykit can also identify clusters of objects using a cylindrical rather than spherical geometry. We implement a parallel version of the algorithm described in Okumura et al. (2017) in the CylindricalGroups class. Our implementation relies on the neighbor querying capability of kdcount and the group-by methods of pandas.

Finally, the FiberCollisions class simulates the process of assigning spectroscopic fibers to objects in a fiberfed redshift survey such as BOSS or eBOSS (Dawson et al. 2013, 2016). This procedure results in so-called "fiber collisions" when two objects are separated by an angular width on the sky that is smaller than the fiber size. We follow the procedure outlined in Guo et al. (2012) to assign fibers to an 
input catalog of objects. We identify angular FOF groups using a linking length equal to the fiber collision scale and assign fibers to the objects in such a way as to minimize the number of objects that do not receive a fiber.

\subsubsection{Miscellaneous}

nbodykit also includes algorithms that generally serve a supporting role in other algorithms. The KDDensity class estimates a proxy density quantity for an input set of objects using the inverse cube of the distance to an object's nearest neighbor. The RedshiftHistogram class computes the mean number density as a function of redshift, $n(z)$, from an input catalog of objects. We plan to generalize this algorithm to be a more universal histogram calculator that could, for example, compute mass or luminosity functions.

\section{Development Workflow}

\subsection{Version Control}

nbodykit is developed using the version control features of $g i t,{ }^{26}$ and the code is hosted in a public repository on GitHub. ${ }^{27}$ Major changes to the code base are performed using a pull request workflow, which provides a mechanism for developers to review changes before they are merged into the main source code. Users can contribute to nbodykit by first forking the main repository, making changes in this fork, and submitting the changes to the main repository via a pull request. This workflow helps assure the overall quality of the code base and ensures that new changes are properly documented and tested. Bugs and new feature requests can be submitted as GitHub issues. As nbodykit is intended as a community-based resource, we encourage user contributions and ideas for new functionality. We adopt a "mentoring" approach for new features and will gladly offer advice and guidance to new users who wish to contribute to nbodykit for the first time.

\subsection{Automated Testing with MPI Support}

nbodykit is extensively tested via hundreds of unit tests using the runtests ${ }^{28}$ package (Feng \& Hand 2017). As mpi4py does not provide a reusable framework for testing parallel applications, we have developed runtests to fill this gap in the development process. It extends the py.test ${ }^{29}$ testing framework, adding several features. First, the test driver incrementally rebuilds and installs the Python package before running the test suite. Second, it adds MPI support by allowing users to specify the number of processes with which each test function should be executed. It also supports computing the testing coverage for parallel applications, where test coverage is defined as the percentage of the software covered by the test suite.

We execute the nbodykit test suite via the continuous integration (CI) service Travis, ${ }^{30}$ using runtests to test both serial and parallel execution of the code. The test suite is currently executed on both Linux and Mac OS X operating systems and for Python versions 2.7, 3.5, and 3.6. Whenever a

\footnotetext{
26 http://git-scm.com

27 http://github.com/bccp/nbodykit

28 https://github.com/rainwoodman/runtests

29 http://pytest.org

30 https://travis-ci.org
}

pull request is opened, the test suite is executed, and the new changes will not be merged if the test suite fails. We also compute the testing coverage of the code base. Currently, nbodykit maintains a value of $95 \%$. We use the Coveralls ${ }^{31}$ service to ensure that new changes cannot be merged into the main repository if the testing coverage decreases.

\subsection{Use on Personal and HPC Machines}

nbodykit is compatible with both Python versions 2.7 and 3.x. For personal computing systems (Mac OS X and Linux), we provide binaries of nbodykit and its dependencies on the Berkeley Center for Cosmological Physics (BCCP) Anaconda channel. ${ }^{32}$ nbodykit (and all of its dependencies) can be installed into an Anaconda environment using a simple command: conda install -c bccp nbodykit. We ensure all packages on the BCCP channel are up to date using a nightly cron job hosted on the Travis CI service.

Supercomputing systems often require recompiling the dependencies of nbodykit using machine-specific compilers and MPI configuration. For example, we use the "conda build" functionality of the Anaconda package to compile and update nbodykit and its dependencies nightly on the NERSC Cray supercomputers. The infrastructure for building nbodykit and its dependencies are publicly available on GitHub, ${ }^{33}$ which users can reuse to set up nbodykit on HPC machines other than NERSC. However, we recommend that users first test whether the default binaries on the BCCP channel are compatible with their supercomputing environment.

The remaining barrier to using nbodykit on HPC systems is the incompatibility of the Python launch system and the shared file systems of HPC machines. When launching an MPI application using Python, the file system will stall when all of the Python instances (can be thousands or more) query the file system for modules on the search path. This issue effectively prevents the use of Python applications on HPC machines.

nbodykit utilizes a lightweight, open-source solution, denoted python-mpi-bcast, to facilitate deploying Python applications on HPC machines (Feng \& Hand 2016). This tool bundles and delivers runtime dependencies to the HPC computing nodes via an MPI broadcast operation, bypassing the file system bottleneck and allowing Python applications to launch at near-native speed. Users can modify their job scripts in a non-invasive manner to deploy our tool. Additional details and setup instructions can be found in Feng \& Hand (2016). The tool is publicly available on GitHub. ${ }^{34}$

Other solutions similar to python-mpi-bcast have been developed to allow the use of Python in HPC environments. Two of the more widely used examples include Shifter ${ }^{35}$ and Spindle. ${ }^{36}$ In our experience, python-mpi-bcast achieves similar results to these tools with less required development overhead.

\footnotetext{
31 https://coveralls.io

32 https://anaconda.org/bccp

33 https://github.com/bccp/conda-channel-bccp

34 https://github.com/rainwoodman/python-mpi-bcast

35 https://github.com/NERSC/shifter

36 https://github.com/hpc/Spindle
} 

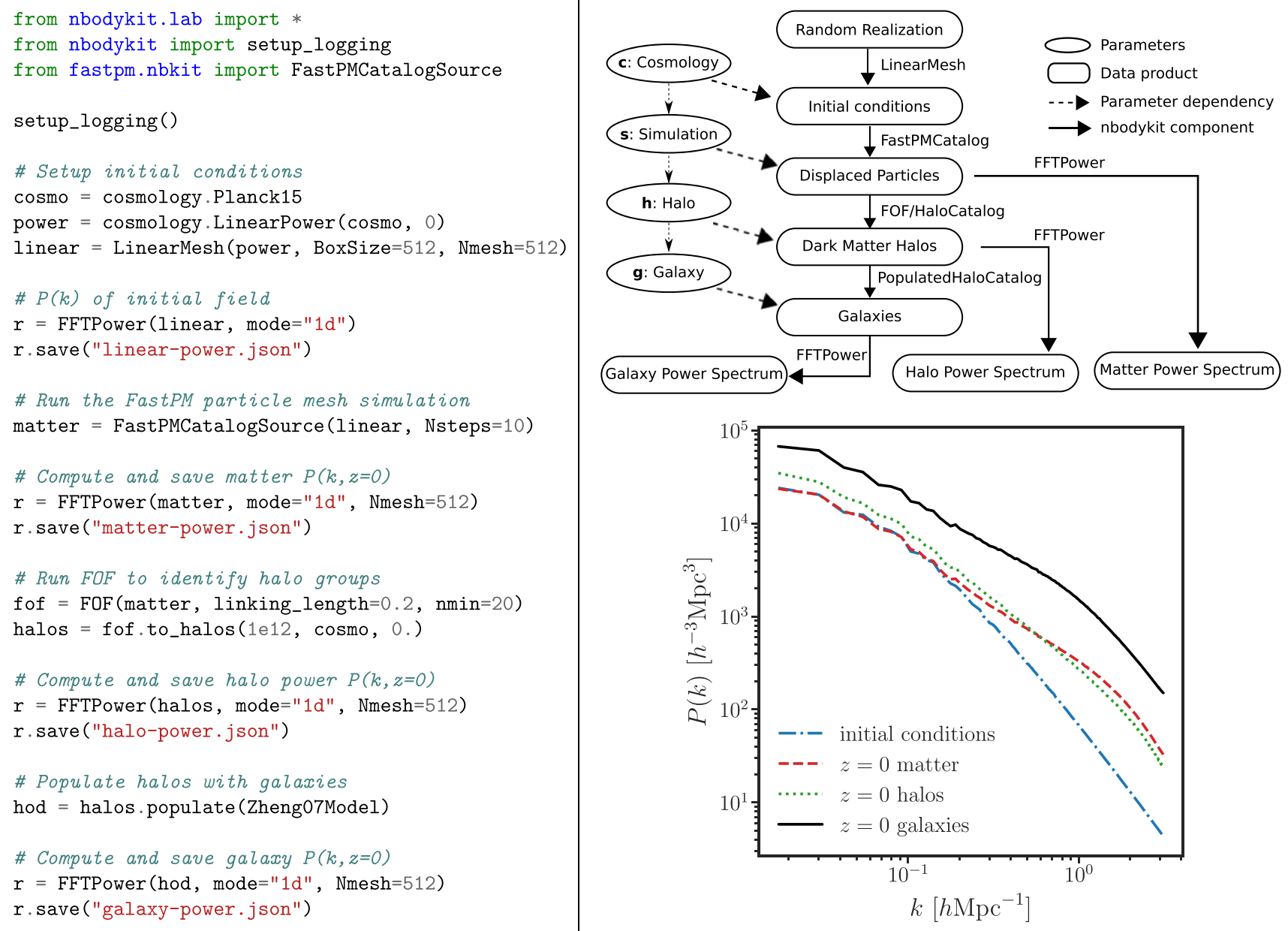

Figure 5. A galaxy clustering emulator, implemented with nbodykit. Left: the source code for the application, which evolves an initial Gaussian field to $z=0$ using the FastPM simulation scheme, identifies FOF halos, populates those halos with galaxies, and records the power spectrum of each step. Right, top: the flow of data through the various components. Right, bottom: the resulting $P(k)$ measured for each step in the emulator. Performance benchmarks for this application are given in Figure 7.

\subsection{Documentation}

Documentation for nbodykit is available on Read the Docs. ${ }^{37}$ The documentation is generated using Sphinx ${ }^{38}$ and includes comprehensive documentation of the nbodykit API. It also includes detailed walkthroughs of each of the main components of nbodykit.

We provide a set of recipes detailing a broad selection of the functionality available in nbodykit in the "Cookbook" section of the documentation. Ranging from simple tasks to more complex workflows, we hope that these recipes help users become acclimated to nbodykit as well as illustrate the power of nbodykit for LSS data analysis. The recipes are in the form of Jupyter notebooks. An interactive environment containing the recipe notebooks is available to users via the Binder service. ${ }^{39}$ This allows new users to explore nbodykit without installing nbodykit on their own machine.

\section{In Action}

In this section, we describe a realistic LSS application using nbodykit: a galaxy clustering emulator. The goal of the

\footnotetext{
37 http://nbodykit.readthedocs.io

38 http://www.sphinx-doc.org

39 https://mybinder.org
}

emulator is to produce the galaxy power spectrum from first principles, given a background cosmological model. The application combines several components of nbodykit to achieve this goal. The steps include

1. Initial conditions: the LinearMesh class creates a Gaussian realization of a density field in Fourier space from an input power spectrum.

2. $N$-body simulation: the initial conditions are evolved forward to $z=0$ using the FastPM quasi- $N$-body particle mesh scheme of Feng et al. (2016).

3. Halo identification: halos are identified from the matter field using the FOF grouping algorithm.

4. Halo population: halos are populated with galaxies using the HOD from Zheng et al. (2007) and the Halotools package.

5. Clustering Estimation: $P(k)$ is computed for each of the above steps using the FFTPower algorithm.

We diagram the flow of data and parameters for these steps in the top-right panel of Figure 5. We also show the source code for the application using nbodykit, which can be implemented using only $\sim 30$ lines of code. We emphasize that with the component-based approach of nbodykit, the user is free to output and serialize any intermediate data products during the execution of the larger application, as we have done 
in this example for the power spectra of the initial, matter, and halo density fields. Finally, note that the source code in Figure 5 can be executed with an arbitrary number of MPI ranks. We discuss performance benchmarks for this application as a function of the number of MPI processes in the next section.

\section{Performance Benchmarks}

In this section, we present performance benchmarks for several nbodykit algorithms, as well as the emulator application discussed in Section 5. Tests are run on the NERSC Cori Phase I Haswell nodes, with 32 MPI cores per node. In Figure 6, we show the strong scaling results for the FFTPower, ConvolvedFFTPower, SimulationBoxPairCount, and SimulationBox3PCF algorithms. The benchmarks are performed for two different data configurations, meant to simulate the data sets of current and future surveys, denoted as "small" and "large," respectively. The "small" sample is modeled after the completed BOSS galaxy sample (Reid et al. 2016) and includes $10^{6}$ galaxies in a cubic box of side length $L=2500 h^{-1} \mathrm{Mpc}$. The "large" sample includes a factor of 10 more objects in a box of side length $L=5000 h^{-1} \mathrm{Mpc}$ and is meant to represent data from future surveys such as DESI (DESI Collaboration et al. 2016). We run four sets of benchmarking tests:

1. FFTPower: compute $P(k, \mu)$ for $10 \mu$ bins, using a mesh size of $N_{\text {mesh }}=1024$. This requires a single FFT operation.

2. ConvolvedFfTPower: compute multipoles $P_{\ell}(k)$ for $\ell=0,2$, and 4 for survey data (R.A., decl., $z$ ), using a mesh size of $N_{\text {mesh }}=1024$. The algorithm requires $2 \ell+1$ FFT operations per multipole and 15 in total for this test.

3. SimulationBoxPairCount: count the number of pairs as a function of separation for 10 separation bins ranging from $r=10 h^{-1} \mathrm{Mpc}$ to $r=150 h^{-1} \mathrm{Mpc}$ and $100 \mu$ bins.

4. SimulationBox3PCF: compute the isotropic 3PCF multipoles for $\ell=0,1, \ldots, 10$ and 10 separation bins ranging from $r=10 h^{-1} \mathrm{Mpc}$ to $r=150 h^{-1} \mathrm{Mpc}$.

In general, these four algorithms show excellent strong scaling with the number of MPI ranks. For the power spectrum algorithms (top row of Figure 6), the dominant calculation is the FFT operation, which has good scaling behavior. Because the FFT is the dominant time cost, we find nearly identical performances for the "small" and "large" samples. The wallclock time for the ConvolvedFFTPower algorithm is roughly 15 times that of the FFTPower algorithm, which is driven by the total number of FFTs that each algorithm computes. The pair-counting-based algorithms both take $\mathcal{O}\left(N^{2}\right)$ time to compute their results. However, the SimulationBoxPairCount algorithm relies on the highly optimized Corrfunc software, which is significantly faster than SimulationBox3PCF, which relies on kdcount. When using SimulationBoxPairCount on the "small" data set, we find that MPI communication costs are non-negligible due to the relatively small sample size, which hinders the scaling performance of the code.

We also present performance benchmarks for the emulator application described in Section 5. For this test, we run a FastPM particle mesh simulation with $512^{3}$ total particles. The

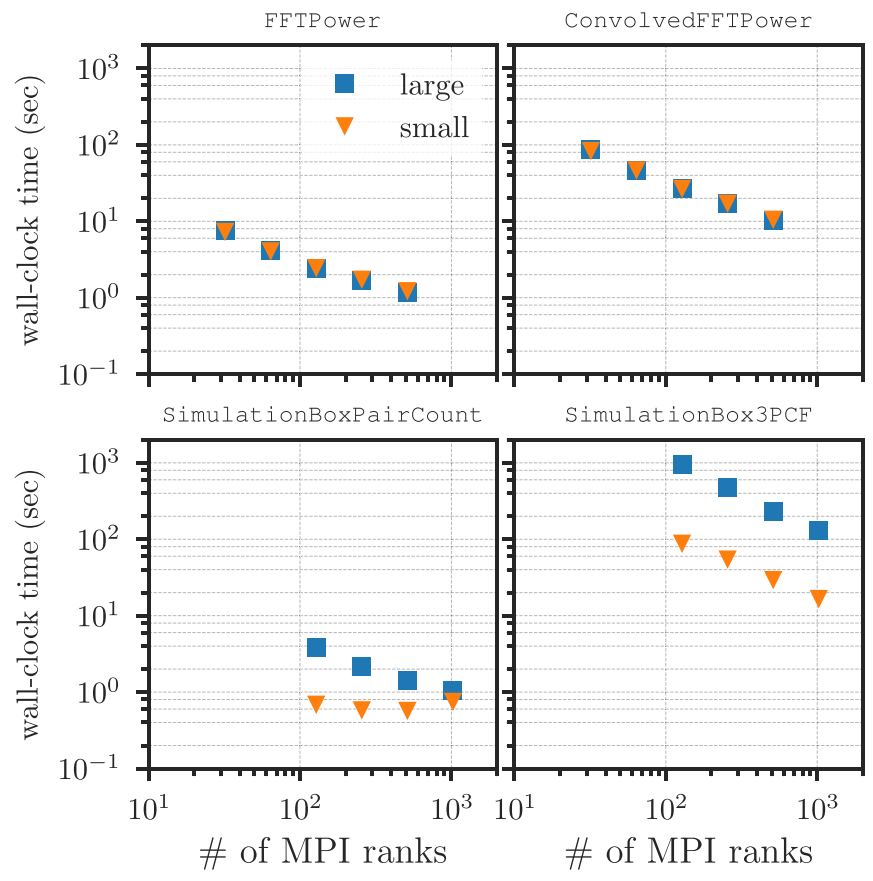

Figure 6. Performance benchmarks for four nbodykit algorithms for our "small" data set $\left(10^{6}\right.$ objects) and our "large" data set $\left(10^{7}\right.$ objects $)$. The algorithms in the top row use FFT-based estimators to compute power spectra, while those in the bottom row of panels count pairs of objects in configuration space. The FFT-based algorithms take near-identical times for the large and small data sets due to the use of a $1024^{3}$ mesh in both cases. The benchmarks were performed on the NERSC Cori Phase I Haswell nodes using 32 MPI ranks per node. See the text of Section 6 for further details on the test configurations.

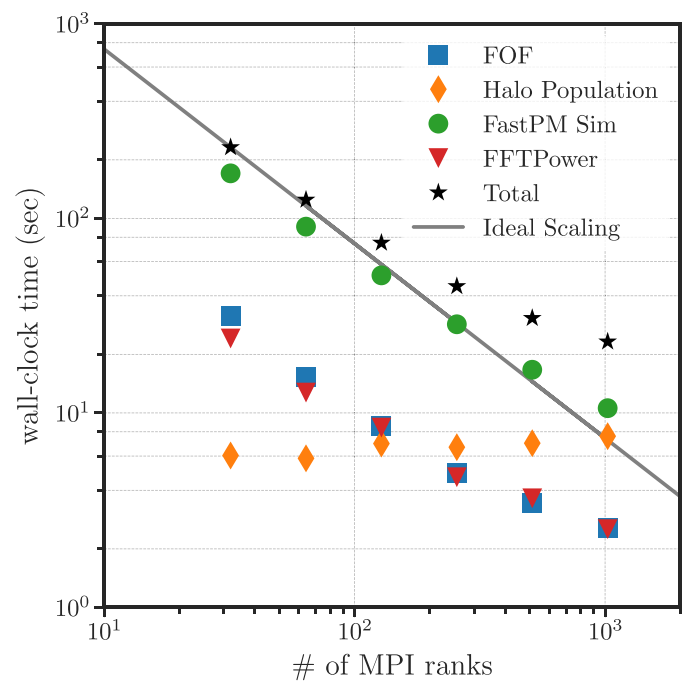

Figure 7. The wall-clock time as a function of the number of MPI ranks used for each step in the galaxy clustering emulator detailed in Figure 5. Overall, the application shows excellent scaling behavior, with deviations from the ideal scaling caused by the halo population step. This step does not currently have a massively parallel implementation and takes a roughly constant amount of time as more cores are used. The benchmarks were performed on the NERSC Cori Phase I Haswell nodes using 32 MPI ranks per node.

halo finder identifies roughly 225,000 dark matter halos that are then used to build a mock galaxy catalog. The wall-clock times for each step in the emulator are shown in Figure 7. We see that the dominant fraction of the wall-clock time is spent in the FastPM step, which shows excellent strong scaling behavior up to the number of cores we have tested. The implementation of 
the halo population step using Halotools is not massively parallel, and therefore, the time to solution for this step remains relatively constant as the number of cores increases. The wallclock time for this step only becomes significant as the number of cores approaches $\sim 1024$, and it would be worth investigating improving this step's scaling if users wish to run often at this scale. However, in our experience, we have not found that the time cost of this step justifies further efforts to convert it to a massively parallel implementation.

We emphasize that for all benchmarks presented in this section, the number of MPI ranks can always be increased such that the time to solution is on the order of seconds. This becomes important for realistic data analyses in LSS, which often require repeating an algorithm's calculation hundreds to thousands of times, e.g., while sampling a parameter space using Markov Chain Monte Carlo or optimization techniques. Due to the availability of large-scale computing resources and the scaling behavior of nbodykit demonstrated here, we believe that nbodykit will be able to meet the computational demands of future LSS data analyses.

\section{Conclusions}

We have presented the first public release of nbodykit (v0.3.0), a massively parallel Python toolkit for the analysis of LSS data. Relying on the mpi 4py binding of MPI, the package includes parallel implementations of a set of canonical algorithms in the field of LSS, including two- and three-point clustering estimators, halo identification and population tools, and quasi- $N$-body simulation schemes. The toolkit also includes a set of distributed data containers that support a variety of data formats common in astronomy, including CSV, FITS, HDF5, binary, and bigfile data. With these tools, we hope nbodykit can serve as a foundation for the community to build upon as we strive to meet the demands of future LSS data sets.

In designing nbodykit, we have attempted to balance the requirements of both a scalable and interactive piece of software. Our ultimate goal was to produce a piece of software that is as usable in a Jupyter notebook environment as on an HPC machine. We have adopted a modular, component-based approach that should enable researchers to integrate nbodykit with their own software to build complicated applications. As an illustration of its power, we have discussed an implementation of a galaxy clustering emulator using nbodykit, which provides a complete forward model for the galaxy power spectrum, starting from an initial, Gaussian density field. We have also demonstrated that the toolkit shows excellent scaling behavior, presenting a set of performance benchmarks for the emulator as well as some of the more commonly used algorithms in nbodykit.

We have outlined our development workflow for producing a piece of reusable scientific software. nbodykit is open source-we strongly believe in the idea of open science and have placed an emphasis on reproducibility when designing nbodykit. Designed for the LSS community, we hope that new users will find nbodykit useful in their own research and that the software can continue to grow and mature in new ways from community feedback and contributions. We are also strong believers in the necessity of unit testing and adequate documentation for open-source tools. We have attempted to meet these goals using the Travis automated testing service and the Read the Docs documentation hosting tools. Finally, we have aimed to make nbodykit widely available and easily installable. The package supports both Python versions 2 and 3, and binary distributions of nbodykit and its dependencies can be installed onto Mac OS X and Linux machines using the Anaconda package manager.

nbodykit currently relies only on MPI for its parallelism. While we have found typical computing environments with 2 GB of memory per core sufficient for current needs, memory use could become a concern in the future. To maximize the number of computing cores per unit memory, we hope to gradually add OpenMP support for parallelization within computing nodes to augment the cross-node parallelization provided by MPI.

In the future, we hope to incorporate the expertise of new developers from both the LSS and Python HPC communities. We expect the knowledge of both communities to be necessary in the data analysis of future surveys. The set of features currently implemented in nbodykit is not meant to be all inclusive but rather a sampling of the more commonly used tools in the field. Most importantly, we hope that nbodykit provides a solid basis for the community to build upon. We warmly welcome feedback and contributions of all forms from the community. As an open-source software, nbodykit was designed as a tool to help the LSS community, and we hope that community contributions can help maximize its benefits for its users.

N.H. and Y.F. thank Martin White for comments on the design of the correlation function algorithms and Manodeep Sinha and Andrew Hearin for coordinating the software interfaces of Corrfunc and Halotools with nbodykit. N.H. and Y.F. thank Rollin Thomas and Lisandro Dalcin for discussions on MPI and Python on massively parallel HPC systems. N.H. and Y.F. thank Matthew Rocklin and Steven Hoyer for discussions on building applications with dask. Y.F. thanks Matthew Turk for insightful discussions about the design of yt. We would also like to thank the communities supporting the open-source software and tools that were invaluable to this work: NumPy, SciPy, pandas, IPython, Jupyter, GitHub, Read the Docs, Travis, and Coveralls. We are grateful for the suite of tools provided by Anaconda, a trademarked Python binary distribution system for scientific computing. We also thank Ray Donnelly and Mike Sarahan of Continuum Analytics, Inc. for their help in building nbodykit binary packages. Finally, we thank the anonymous referee for comments improving this work.

In addition, a large number of researchers in the field of cosmology provided useful feedback and input on the development of nbodykit: Man-yat Chu, Biwei Dai, Zhejie Ding, Lukas Heizmann, Zvonimir Vlah, Elena Massara, Mehdi Rezaie, Marcel Schmittful, Hee-Jong Seo, and Miguel Zumalacárregui.

This work used resources of the National Energy Research Scientific Computing Center, a DOE Office of Science User Facility supported by the Office of Science of the U.S. Department of Energy under contract No. DE-AC0205CH11231. N.H. is supported by the U.S. Department of Energy, Office of Science, Office of Workforce Development for Teachers and Scientists, Office of Science Graduate Student Research (SCGSR) program. The SCGSR program is administered by the Oak Ridge Institute for Science and Education for the DOE under contract number DE-SC0014664. Support for 
this work was also provided by the National Aeronautics and Space Administration through Einstein Postdoctoral Fellowship Award Number PF7-180167 issued by the Chandra X-ray Observatory Center, which is operated by the Smithsonian Astrophysical Observatory for and on behalf of the National Aeronautics Space Administration under contract NAS803060. Z.S. also acknowledges support from a Chamberlain Fellowship at Lawrence Berkeley National Laboratory (held previously to the Einstein) and from the Berkeley Center for Cosmological Physics. F.B. acknowledges support by an STFC Ernest Rutherford Fellowship, grant reference ST/P004210/1.

Software: Astropy (Astropy Collaboration et al. 2013; The Astropy Collaboration et al. 2018), bigfile (Feng 2017c), classylss (Hand \& Feng 2017), Corrfunc (Sinha \& Garrison 2017), Dask (Dask Development Team 2016), fastpm (Feng 2017e), fitsio (Sheldon 2017), h5py (Collette 2017), Halotools (Hearin et al. 2017), IPython (Perez \& Granger 2007), Jupyter (Kluyver et al. 2016), kdcount (Feng 2017d), mpi4py (Dalcín et al. 2008), Numpy (van der Walt et al. 2011), Pandas (McKinney 2010), pfft-python (Feng 2017a), pmesh (Feng 2017b), pytest (Krekel et al. 2004), runtests (Feng \& Hand 2017), Scipy (Jones et al. 2001-2017).

\section{ORCID iDs}

Nick Hand (ib https://orcid.org/0000-0002-8809-3939

\section{References}

Agrawal, A., Makiya, R., Chiang, C.-T., et al. 2017, JCAP, 10, 003 Alam, S., Ata, M., Bailey, S., et al. 2017, MNRAS, 470, 2617 Astropy Collaboration, Robitaille, T. P., Tollerud, E. J., et al. 2013, A\&A, 558, A33

Behroozi, P. S., Wechsler, R. H., \& Wu, H.-Y. 2013, ApJ, 762, 109 Beutler, F., Saito, S., Brownstein, J. R., et al. 2014, MNRAS, 444, 3501

Beutler, F., Seo, H.-J., Saito, S., et al. 2017, MNRAS, 466, 2242

Bianchi, D., Gil-Marín, H., Ruggeri, R., \& Percival, W. J. 2015, MNRAS, 453, L11

Blake, C., \& Glazebrook, K. 2003, ApJ, 594, 665

Blas, D., Lesgourgues, J., \& Tram, T. 2011, JCAP, 7, 034

Castorina, E., \& White, M. 2018, MNRAS, 476, 4403

Cole, S., Fisher, K. B., \& Weinberg, D. H. 1995, MNRAS, 275, 515

Cole, S., Percival, W. J., Peacock, J. A., et al. 2005, MNRAS, 362, 505

Coles, P., \& Jones, B. 1991, MNRAS, 248, 1

Collette, A. 2017, HDF5 for Python, http://www.h5py.org

Cui, W., Liu, L., Yang, X., et al. 2008, ApJ, 687, 738

Dalcín, L., Paz, R., Storti, M., \& DElía, J. 2008, JPDC, 5, 655

Dalcin, L. D., Paz, R. R., Kler, P. A., \& Cosimo, A. 2011, AdWR, 34, 1124

Dask Development Team 2016, Dask :Library for Dynamic Task Scheduling, http://dask.pydata.org

Daubechies, I. 1992, Ten Lectures on Wavelets (Philadelphia, PA: SIAM)

Davis, M., Efstathiou, G., Frenk, C. S., \& White, S. D. M. 1985, ApJ, 292, 371

Dawson, K. S., Kneib, J.-P., Percival, W. J., et al. 2016, AJ, 151, 44

Dawson, K. S., Schlegel, D. J., Ahn, C. P., et al. 2013, AJ, 145, 10

Delubac, T., Bautista, J. E., Busca, N. G., et al. 2015, A\&A, 574, A59

DESI Collaboration, Aghamousa, A., Aguilar, J., et al. 2016, arXiv:1611. 00036

Desjacques, V., \& Seljak, U. 2010, CQGra, 27, 124011

Diemer, B. 2017, arXiv:1712.04512

Ding, Z., Seo, H.-J., Vlah, Z., et al. 2018, MNRAS, 479, 1021

Efstathiou, G., Sutherland, W. J., \& Maddox, S. J. 1990, Natur, 348, 705

Eisenstein, D. J., \& Hu, W. 1999, ApJ, 511, 5

Eisenstein, D. J., Hu, W., \& Tegmark, M. 1998, ApJL, 504, L57

Eisenstein, D. J., Zehavi, I., Hogg, D. W., et al. 2005, ApJ, 633, 560

Everitt, B. S., Landau, S., \& Leese, M. 2009, Cluster Analysis (4th ed.; New York: Wiley)

Feldman, H. A., Kaiser, N., \& Peacock, J. A. 1994, ApJ, 426, 23

Feng, Y. 2017a, pfft-python, v0.1.13, Zenodo, doi:10.5281/zenodo.1051308

Feng, Y. 2017b, pmesh, v0.1.33, Zenodo, doi:10.5281/zenodo.1051254

Feng, Y. 2017c, bigfile, v0.1.39, Zenodo, doi:10.5281/zenodo.1051252
Feng, Y. 2017d, kdcount, v0.2.9, Zenodo, doi:10.5281/zenodo.1051244

Feng, Y. 2017e, fastpm-python, v0.0.6, Zenodo, doi:10.5281/zenodo.1051310

Feng, Y., Chu, M.-Y., Seljak, U., \& McDonald, P. 2016, MNRAS, 463, 2273

Feng, Y., \& Hand, N. 2016, in Proc. 15th Python in Science Conf., ed. S. Benthall \& S. Rostrup, 137

Feng, Y., \& Hand, N. 2017, runtests, v0.0.23, Zenodo, doi:10.5281/zenodo. 1051306

Feng, Y., \& Modi, C. 2017, A\&C, 20, 44

Font-Ribera, A., Kirkby, D., Busca, N., et al. 2014, JCAP, 5, 027

Friesen, B., Patwary, M. M. A., Austin, B., et al. 2017, in Proc. Int. Conf. for High Performance Computing, Networking, Storage and Analysis, SC '17 (New York: ACM), 1, http://doi.acm.org/10.1145/3126908.3126927

Guo, H., Zehavi, I., \& Zheng, Z. 2012, ApJ, 756, 127

Hamilton, A. J. S. 2000, MNRAS, 312, 257

Hand, N., \& Feng, Y. 2017, classylss, v0.2.7, Zenodo, doi:10.5281/zenodo. 1051256

Hand, N., Li, Y., Slepian, Z., \& Seljak, U. 2017a, JCAP, 7, 002

Hand, N., Seljak, U., Beutler, F., \& Vlah, Z. 2017b, JCAP, 10, 009

Hearin, A. P., Campbell, D., Tollerud, E., et al. 2017, AJ, 154, 190

Hearin, A. P., Zentner, A. R., van den Bosch, F. C., Campbell, D., \& Tollerud, E. 2016, MNRAS, 460, 2552

Hinshaw, G., Larson, D., Komatsu, E., et al. 2013, ApJS, 208, 19

Hockney, R. W., \& Eastwood, J. W. 1981, Computer Simulation Using Particles (New York: McGraw-Hill)

Jain, A., Topchy, A., Law, M., \& Buhmann, J. 2004, in Proc. Int. Conf. Pattern Recognition 1, ed. J. Kittler, M. Petrou, \& M. Nixon (Cambridge: IEEE), 260 Jarvis, M., Bernstein, G., \& Jain, B. 2004, MNRAS, 352, 338

Jones, E., Oliphant, T., Peterson, P., et al. 2001-2017, SciPy: Open source scientific tools for Python, http://www.scipy.org/

Kluyver, T., Ragan-Kelley, B., Pérez, F., et al. 2016, in Positioning and Power in Academic Publishing: Players, Agents and Agendas, ed. F. Loizides \& B. Schmidt (IOS Press), 87

Komatsu, E., Dunkley, J., Nolta, M. R., et al. 2009, ApJS, 180, 330

Komatsu, E., Smith, K. M., Dunkley, J., et al. 2011, ApJS, 192, 18

Krauss, L. M., \& Turner, M. S. 1995, GReGr, 27, 1137

Krekel, H., Oliveira, B., Pfannschmidt, R., et al. 2004, pytest 3.6.4, https:// github.com/pytest-dev/pytest

Landy, S. D., \& Szalay, A. S. 1993, ApJ, 412, 64

Leauthaud, A., Tinker, J., Behroozi, P. S., Busha, M. T., \& Wechsler, R. H 2011, ApJ, 738, 45

Lesgourgues, J. 2011, arXiv:1104.2932

Lesgourgues, J., \& Pastor, S. 2006, PhR, 429, 307

Lewis, A., Challinor, A., \& Lasenby, A. 2000, ApJ, 538, 473

Maddox, S. J., Efstathiou, G., Sutherland, W. J., \& Loveday, J. 1990, MNRAS, 242, 43P

McKinney, W. 2010, in Proc. 9th Python in Science Conf., ed. S. van der Walt \& J. Millman, 51

Merz, H., Pen, U.-L., \& Trac, H. 2005, NewA, 10, 393

Modi, C., Castorina, E., \& Seljak, U. 2017, MNRAS, 472, 3959

Momcheva, I., \& Tollerud, E. 2015, arXiv:1507.03989

Moore, A. W., Connolly, A. J., Genovese, C., et al. 2001, in Mining the Sky: Proc. of the MPA/ESO/MPE Workshop, ed. A. J. Banday, S. Zaroubi, \& M. Bartelmann (Berlin: Springer-Verlag), 71

Mueller, E.-M., Percival, W., Linder, E., et al. 2018, MNRAS, 475, 2122

NSF 2017, NSF Committee on Software Infrastructure for Heterogeneous Computing, https://github.com/labarba/NSFcommittee-SI2017/

Okumura, T., Takada, M., More, S., \& Masaki, S. 2017, MNRAS, 469, 459

Ostriker, J. P., \& Steinhardt, P. J. 1995, Natur, 377, 600

Perez, F., \& Granger, B. E. 2007, CSE, 9, 21

Perlmutter, S., Aldering, G., Goldhaber, G., et al. 1999, ApJ, 517, 565

Pinol, L., Cahn, R. N., Hand, N., Seljak, U., \& White, M. 2017, JCAP, 4, 008

Pippig, M. 2013, SIAM Journal on Scientific Computing, 35, C213

Planck Collaboration, Ade, P. A. R., Aghanim, N., et al. 2014, A\&A, 571, A16 Planck Collaboration, Ade, P. A. R., Aghanim, N., et al. 2016, A\&A, 594, A13

Reid, B., Ho, S., Padmanabhan, N., et al. 2016, MNRAS, 455, 1553

Riess, A. G., Filippenko, A. V., Challis, P., et al. 1998, AJ, 116, 1009

Schmittfull, M., Baldauf, T., \& Zaldarriaga, M. 2017, PhRvD, 96, 023505

Scoccimarro, R. 2015, PhRvD, 92, 083532

Sefusatti, E., Crocce, M., Scoccimarro, R., \& Couchman, H. M. P. 2016, MNRAS, 460, 3624

Seo, H.-J., \& Eisenstein, D. J. 2003, ApJ, 598, 720

Sheldon, E. 2017, A Python Package for FITS Input/Output Wrapping Cfitsio, https://github.com/esheldon/fitsio

Sinha, M., \& Garrison, L. 2017, Corrfunc: Blazing fast correlation functions on the CPU, Astrophysics Source Code Library, ascl:1703.003

Slepian, Z., \& Eisenstein, D. J. 2015a, arXiv:1510.04809 
Slepian, Z., \& Eisenstein, D. J. 2015b, MNRAS, 454, 4142

Slepian, Z., \& Eisenstein, D. J. 2016, MNRAS, 455, L31

Slepian, Z., \& Eisenstein, D. J. 2018, MNRAS, 478, 1468

Slepian, Z., Eisenstein, D. J., Brownstein, J. R., et al. 2017, MNRAS, 469, 1738

Slosar, A., Hirata, C., Seljak, U., Ho, S., \& Padmanabhan, N. 2008, JCAP, 8,031

Smith, R. E., Peacock, J. A., Jenkins, A., et al. 2003, MNRAS, 341, 1311

Springel, V. 2005, MNRAS, 364, 1105

Springel, V., Yoshida, N., \& White, S. D. M. 2001, NewA, 6, 79

Takahashi, R., Sato, M., Nishimichi, T., Taruya, A., \& Oguri, M. 2012, ApJ, 761,152

Tassev, S., Zaldarriaga, M., \& Eisenstein, D. J. 2013, JCAP, 6, 036
The Astropy Collaboration, Price-Whelan, A. M., Sipőcz, B. M., et al. 2018, arXiv:1801.02634

Turk, M. J., Smith, B. D., Oishi, J. S., et al. 2011, ApJS, 192, 9

van der Walt, S., Colbert, S. C., \& Varoquaux, G. 2011, CSE, 13, 22

Vlah, Z., Seljak, U., \& Baldauf, T. 2015, PhRvD, 91, 023508

Waters, D., Di Matteo, T., Feng, Y., Wilkins, S. M., \& Croft, R. A. C. 2016 MNRAS, 463, 3520

White, M. 2002, ApJS, 143, 241

White, M., Tinker, J. L., \& McBride, C. K. 2014, MNRAS, 437, 2594

Yamamoto, K., Nakamichi, M., Kamino, A., Bassett, B. A., \& Nishioka, H. 2006, PASJ, 58, 93

Yang, Y.-B., Feng, L.-L., Pan, J., \& Yang, X.-H. 2009, RAA, 9, 227

Zheng, Z., Coil, A. L., \& Zehavi, I. 2007, ApJ, 667, 760 